\title{
Visual paired comparison performance is impaired in a patient with selective hippocampal lesions and relatively intact item recognition
}

\author{
O. Pascalis ${ }^{\text {a,* }}$, N. M. Hunkin ${ }^{\text {a }}$, J. S. Holdstock ${ }^{\text {b }}$, C. L. Isaac ${ }^{\text {a }}$, A. R. Mayes ${ }^{\text {b }}$ \\ a LGF Group, Department of Psychology, The University of Sheffield, Western Bank, Sheffield S10 2TP, UK \\ ${ }^{\mathrm{b}}$ Department of Psychology, University of Liverpool, Eleanor Rathbone Building, Bedford Street South, Liverpool L69 3BS, UK
}

Received 16 July 2003; accepted 16 March 2004

\begin{abstract}
In this study, we have examined visual recognition memory in a patient, YR, with discrete hippocampal damage who has shown normal or nearly normal item recognition over a large number of tests. We directly compared her performance as measured using a visual paired comparison task (VPC) with her performance on delayed matching to sample (DMS) tasks. We also investigated the effect of retention interval between familiarisation and test. YR shows good visual recognition with the DMS task up to $10 \mathrm{~s}$ after the familiarisation period, but only shows recognition with the VPC task for the shortest retention interval $(0 \mathrm{~s})$. Our results are consistent with the view that hippocampal damage disrupts recollection and recall, but not item familiarity memory.

(C) 2004 Elsevier Ltd. All rights reserved.
\end{abstract}

Keywords: Recognition memory; Hippocampus; Amnesia; VPC

\section{Introduction}

Evidence from lesion studies indicates that damage to the medial temporal lobes impairs visual recognition memory and episodic memory (Squire, 1992). There is consistent evidence from studies in monkeys that a visual item recognition memory deficit occurs after damage to medial temporal cortex sites that are adjacent to the hippocampal formation. Selective lesions of either the entorhinal and perirhinal cortex (Gaffan \& Murray, 1992), or the perirhinal cortex and parahippocampal cortex (Zola-Morgan, Squire, Amaral, $\&$ Suzuki, 1989) or the perirhinal cortex alone (Meunier, Bachevalier, Mishkin, \& Murray, 1993) yielded severe recognition memory loss. There is, however, conflicting evidence on the effects of selective hippocampal lesions on visual item recognition memory and disagreement about the mnemonic role played by the hippocampus. Whereas, some animal lesion studies, using the Delayed Non-Matching to Sample (DNMS) task, have found that selective damage to the hippocampal formation resulted in impairment at the longest delays only (Zola-Morgan, Squire, Rempel, Clower, \& Amaral, 1992; Alvarez, Zola-Morgan, \& Squire, 1995), other studies have found no impairment of visual recognition memory ability (Murray \& Mishkin, 1998). The effect

\footnotetext{
* Corresponding author. Tel.: +44-114-222-6548.

E-mail address: o.pascalis@sheffield.ac.uk (O. Pascalis).
}

of a hippocampal lesion on visual recognition memory as measured with the DNMS task is then still under debate (see Bachevalier, Nematic, \& Alvarado, 2003; Baxter \& Murray, 2001a for a discussion).

Human lesion studies have found similar conflicting results. Whereas, some studies have found clear visual item recognition deficits after relatively selective hippocampal damage (e.g., Cipolotti et al., 2001; Manns \& Squire, 1999; Manns, Hopkins, Reed, Kitchener, \& Squire, 2003; Reed $\&$ Squire, 1997), others have found little or no impairment in item recognition memory (Mayes, Holdstock, Isaac, Hunkin, \& Roberts, 2002; Vargha-Khadem et al., 1997; Yonelinas et al., 2002). The explanation of these conflicting results is unknown, but most probably involves either differing extents and locations of hippocampal damage, differing extents of damage or dysfunction in extra-hippocampal sites critical for visual item recognition, or both (for example, see Baxter \& Murray, 2001a and b; Zola \& Squire, 2001).

Aggleton and Brown (1999) have hypothesised that damage restricted to the hippocampal formation impairs only recollection, leaving familiarity-based item recognition memory intact. The evidence about whether hippocampal damage leaves familiarity memory intact is conflicting. Whereas, Yonelinas et al. (2002) found, using several methods of assessment, that familiarity was preserved in patients who probably suffered hippocampal damage caused by hypoxia following a cardiac arrest, Manns et al. (2003) found that 
familiarity was as impaired as recollection in a group of patients who had suffered hippocampal damage. Provided, as many researchers assume, that relatively normal item recognition memory can be supported by familiarity alone, the Aggleton and Brown hypothesis is consistent with hippocampal lesions causing only a mild item recognition deficit that might not be detectable in single patients. In contrast, if hippocampal lesions disrupt familiarity as well as recollection, then they should severely disrupt item recognition memory.

Item recognition memory has been assessed not only directly through the use of tests such as DNMS, but also indirectly with the Visual Paired Comparison (VPC) task (McKee \& Squire, 1993; Pascalis \& Bachevalier, 1999; Zola et al., 2000). The VPC task, which was developed by Fantz (1964), is a common way to measure visual recognition in preverbal and nonverbal individuals (Fagan, 1974; Pascalis \& Bachevalier, 1998). It exploits individuals' attraction to novelty in order to assess their recognition memory for previously seen stimuli. The basic procedure is as follows: The participant is first presented with a stimulus for a familiarisation period. Later, the participant is presented with the same stimulus paired with a novel one. The key measure is the length of time spent fixating each of the two stimuli. Longer duration of looking towards one stimulus, generally the novel one, indicates discrimination and, indirectly, recognition memory. Long-term recognition memory has been shown during infancy with this task (Fagan, 1974; Pascalis, de Haan, Nelson, \& de Schonen, 1998). The type of memory assessed by this task in infants has been controversial, but according to Nelson (1995), it tests a pre-explicit form of memory. Recently, however, a study of adults showed that novelty preference correlated with later recognition of the non-preferred stimulus, which is consistent with the view that it provides an indirect index of the ability to show aware recognition of studied stimuli (Manns, Stark, \& Squire, 2000).

Performance on the VPC task, in both infant and adult monkeys with damage to the medial temporal lobe that included the hippocampal formation, amygdala and surrounding tissue has been found to be abnormal (Bachevalier, Brickson, \& Hagger, 1993). Recently, Pascalis and Bachevalier (1999) showed that adult monkeys with neonatal hippocampal lesions showed preference for novelty at short delays of $10 \mathrm{~s}$, but not at longer delays of $30 \mathrm{~s}$ to $24 \mathrm{~h}$, whereas normal monkeys showed novelty preference at all delays. Consistent with this study, other studies of monkeys with selective lesions within the medial temporal lobe, have shown that novelty preference depends on the integrity of the hippocampal formation (Zola et al., 2000) as well as the perirhinal cortex (Buffalo et al., 1999). In humans, McKee and Squire (1993), using the VPC task, have shown that amnesic patients with relatively selective hippocampal damage also show abnormal novelty preference. The patients in this study also showed clearly impaired visual item recognition when this was measured directly, although their performance was above chance levels.
Although monkeys, who showed an impaired novelty preference on the VPC task (Pascalis \& Bachevalier, 1999), showed close to normal recognition on a DNMS task (Bachevalier, Beauregard, \& Alvarado, 1999), this has not been demonstrated in any human patients with relatively selective hippocampal lesions. If a single dissociation can be shown between VPC performance and a direct performance measure of visual item recognition, this should help constrain hypotheses about the processes that underlie directly measured recognition and VPC performance. To determine whether this kind of single dissociation can be found in humans, we have examined VPC performance in a patient, YR, with discrete hippocampal damage who has shown normal or nearly normal item recognition over a large number of tests (Mayes et al., 2002). We directly compared her VPC performance with her performance on delayed matching to sample (DMS) tasks. We also investigated the effect of retention interval between familiarisation and test.

\section{Methods}

\subsection{Participants}

The participants were patient YR and five age- and IQ-matched healthy control participants. YR, a female, was 62-year-old at the time of testing and had developed a memory impairment following a possible ischaemic infarct, arising from the administration of an opiate drug to relieve severe back pain 14 years previously. YR's neuropathology and neuropsychological profile are reported in detail by Holdstock et al. (2000) and Mayes et al., in press. For clarification, an overview of YR's neuropathological and neuropsychological details are included here.

Magnetic resonance imaging (MRI) was carried out in September 1997 using a 1.5 T SIGNA whole-body magnetic imaging system (General Electric, Milwaukee, WI). A 3D T1-weighted radio-frequency spoiled gradient echo (SPGR) image revealed a selective lesion affecting the hippocampus bilaterally along its full anterior-posterior extent. Volumetric analysis indicated that the volumes of the hippocampi (corrected for intracranial volume) were 2.5 and 3 S.D.s smaller than the mean volumes in a group of healthy control participants (matched for sex, age and IQ) on the right and left, respectively. In contrast, there was no pathology evident in the parahippocampal gyrus, and the corrected volume of this region, which included the perirhinal, entorhinal and parahippocampal cortices, was at least 1 S.D. greater than that of the control participants. Although the amygdala appeared small, there was no evidence of pathology. Frontal lobe structures were intact, and grey to white matter ratios were normal. There was some evidence of parietal lobe atrophy, but this was not atypical for a woman of YR's age, and her corrected parietal lobe volume was within the control range on the right, and only just below the control range on the left. 
Table 1

Performance of YR on standardised tests of intellectual and memory function

\begin{tabular}{llr}
\hline Tests & Subtests & YR's performance \\
\hline NART FSIQ & & 115 \\
WAIS-R & Full & 102 \\
& Verbal & 108 \\
& Performance & 97 \\
WMS-R & Verbal & 62 \\
& Visual & 102 \\
& General & 66 \\
& Attention & 122 \\
& Delay & 73 \\
WRMT & Words & $11^{\mathrm{a}}(50-75$ percentile $)$ \\
& Faces & $16^{\mathrm{a}}(>95$ percentile $)$ \\
D\&P & People (verbal recall) & $4^{\mathrm{a}}(<5$ percentile $)$ \\
& Names (verbal recognition) & $16^{\mathrm{a}}(99$ percentile) \\
& Shapes (non-verbal recall) & $5^{\mathrm{a}}(1-5$ percentile $)$ \\
& Doors (non-verbal recognition) & $9^{\mathrm{a}}(50$ percentile) \\
\hline
\end{tabular}

Key: NART FSIQ: estimated full scale IQ from National Adult Reading Test (Nelson, 1991); WAIS-R: Wechsler Adult Intelligence Scale, Revised (Wechsler, 1981); WMS-R: Wechsler Memory Scales, Revised (Wechsler, 1987); WRMT: Warrington Recognition Memory Scale (Warrington, 1984); D\&P: Doors and People Test (Baddeley et al., 1994).

${ }^{a}$ Denotes age-scaled scores.

YR's performance on standardised tests of IQ and memory function given up to the time of scanning are shown in Table 1. Her current FSIQ (WAIS-R; Wechsler, 1981) was in the average range and, although she showed a decrement of 13 points between her estimated pre-morbid IQ (NART; Nelson, 1991) and her current IQ, this represented a drop of $<1$ S.D. in IQ points. On tests of memory function, YR showed a consistent pattern in that she had a severe impairment on tests of verbal and non-verbal recall, but intact performance on tests of verbal and non-verbal item recognition (see Mayes et al., 2002). This pattern of impairment is shown clearly by her performance on the Doors and People Test (Baddeley, Emslie, \& Nimmo-Smith, 1994) on which she scored below the fifth percentile on tests of recall, but at the 50th percentile or above on tests of recognition. Her performance on the verbal and visual subtests of the Warrington Recognition Memory Test (WRMT; Warrington, 1984) was also above the 50th percentile in both cases. The dissociation between YR's impaired visual as well as verbal recall and her relatively intact performance on tests of visual and verbal item recognition respectively has been studied in greater detail, and is reported by Mayes et al. (2002).

In addition to tests of memory, YR's perceptual function was assessed with the Visual Object and Space Perception Battery (VOSP; Warrington \& James, 1991). YR scored within 1 S.D. of the mean of the normative sample for all subtests, and performed better than the controls on one of the four object perception subtests (Silhouettes) and three of the four spatial subtests (Dot Counting, Position Discrimination, Cube Analysis).
Five control participants were recruited. These were healthy, female volunteers who were matched to YR in terms of age (mean age: 63.20 years, S.D.: 2.17) and WAIS-R FSIQ (mean IQ: 101.60, S.D.: 5.03).

\subsection{Stimuli}

Different sets of stimuli were used in the VPC and DMS tasks.

\subsubsection{VPC task}

One hundred and sixty black and white slides of objects and faces were used as stimuli. The size and brightness of the objects were kept uniform on each slide. When projected onto the screen, the size of the stimuli was $8 \mathrm{~cm} \times 10 \mathrm{~cm}$, and when two stimuli were present, they were separated by a $5 \mathrm{~cm}$ gap.

\subsubsection{Delayed matching to sample task (DMS)}

2.2.2.1. Objects. Seventy-two colour photographs of everyday objects were selected. The photographs were presented on a computer screen to eight young healthy volunteers, who were required to rate each photograph on a scale of familiarity from 1 to 5 . The 72 photographs were then divided into six sets of 12 , which were matched on the familiarity rating. Three of the sets were designated 'target' sets and three were designated 'distractor' sets; each target set was paired with a distractor set. Each pair of sets was allocated to one of the three delay conditions $(0,5,10 \mathrm{~s}$ delay). The photographs were saved as black and white pictures, and were presented on a white background. All pictures occupied a $7 \mathrm{~cm} \times 8.5 \mathrm{~cm}$ rectangle on the computer screen. During study, single pictures were presented at the centre of the screen. At test, two pictures were presented side-by-side separated by a $5.5 \mathrm{~cm}$ gap.

2.2.2.2. Faces. Seventy-two black and white novel faces were selected from our database. The 72 faces were arranged into 36 pairs, each of which comprised two faces that were matched in gender, approximate age and physical appearance. One member of each pair was designated the target face, and the other was designated the distractor face. The 36 pairs were divided into three sets of 12 pairs. Each set was allocated to one of the three delay conditions $(0,5,10 \mathrm{~s}$ delay). All faces were presented on a white background, and occupied a $12 \mathrm{~cm} \times 12 \mathrm{~cm}$ rectangle on the computer screen. During study, single pictures were presented at the centre of the screen. At test, two pictures were presented side-by-side separated by a $2 \mathrm{~cm}$ gap.

\subsection{Procedure}

The two tasks were administered separately over several sessions. The VPC task was administered first during four 
sessions over a 1 month period. The DMS task was administered in one session 2 months later.

\subsubsection{VPC task}

YR and the controls were investigated individually. Experimental conditions were as follows. First, each participant was shown a single target stimulus to inspect during a $5 \mathrm{~s}$ familiarisation period. After a delay during which a blank screen was presented, the participant was shown the target object or face paired with a new stimulus, for $5 \mathrm{~s}$. The left-right position of the novel stimulus was counterbalanced across trials. The delays tested were no delay (i.e., the time taken to change the slide, which was around $1 \mathrm{~s}$ ), 5 and $10 \mathrm{~s}$. Twelve trials with faces and 12 trials with objects were used at each delay. The trials for each delay and category of stimulus were randomly intermixed and presented over several testing sessions. Participants were told that they were part of a vision study, and we explained to YR that she was the control of a patient with a visual problem. Participants were instructed that one picture would appear on the screen for a brief presentation followed by a brief period of rest, then two pictures would be simultaneously presented. They were asked only to "look at the screen as if you were watching TV". The dependent variable was actual looking time directed to the new and to the old stimulus.

A video camera with a videotimer was fixed above the screen and recorded participants' eye movements onto videotape. Stimulus fixation was indicated by corneal reflection of the stimuli. Inspection of the videotape after the experiment allowed the time spent inspecting the right and left images in the $5 \mathrm{~s}$ recognition phase to be assessed.

\subsubsection{DMS task}

Each participant was exposed to a stimulus for $5 \mathrm{~s}$ and instructed to remember it. After a brief delay $(0,5$ or $10 \mathrm{~s})$, during which a blank screen was presented, the participant was shown the familiar (target) stimulus together with a novel (distractor) stimulus. The participant was required to point to the familiar stimulus. The two stimuli were presented side-by-side, and the left/right position of the familiar/novel stimuli was counterbalanced across trials. There were 12 trials at each of the three delays for objects and 12 trials at each of the three delays for faces. Each delay condition was run separately in the following order: objects 0,5 , $10 \mathrm{~s}$; faces $0,5,10 \mathrm{~s}$. The order of conditions was the same for all participants. Within each condition, the stimuli were presented in random order.

\section{Results}

To determine whether there was a significant difference between the novelty preference of YR and that of the control participants on the VPC task, the difference in time spent looking at the familiar and novel stimuli was analysed. Novelty preference was defined as the time spent looking at the
Table 2

Mean difference in time spent looking at novel and familiar pictures

\begin{tabular}{cllll}
\hline Condition & $\begin{array}{l}\text { Control mean } \\
\text { (S.D.) }\end{array}$ & $\begin{array}{l}\text { YR mean } \\
\text { (S.D.) }\end{array}$ & $t$-value & $\begin{array}{l}\text { Probability } \\
\text { (two-tailed) }\end{array}$ \\
\hline $\begin{array}{llll}\text { Objects } \\
0 \mathrm{~s}\end{array}$ & $1.030(1.275)$ & $0.900(1.258)$ & 0.964 & 0.349 \\
$5 \mathrm{~s}$ & $1.276(1.552)$ & $-0.487(2.173)$ & 4.456 & 0.001 \\
$10 \mathrm{~s}$ & $1.415(1.393)$ & $-0.200(1.928)$ & 5.185 & 0.000 \\
& & & & \\
Faces & & $1.033(1.138)$ & 1.873 & 0.078 \\
$0 \mathrm{~s}$ & $1.241(1.216)$ & $0.325(1.257)$ & 7.859 & 0.000 \\
$5 \mathrm{~s}$ & $1.404(1.532)$ & $0.453(1.357)$ & 7.677 & 0.000 \\
$10 \mathrm{~s}$ & $1.669(1.534)$ & & & \\
\hline
\end{tabular}

A positive value indicate a novelty preference.

familiar stimulus subtracted from the time spent looking at the novel stimulus. This was calculated for each participant on each of the 12 trials in each condition. YR's novelty preference in each condition was then compared with that of the control participants using a $t$-test. Since it cannot be assumed that the variance of YR's performance was equivalent to that of the group of control participants, Welch's procedure, which tests for the significance of the difference between means when the population variances are unequal (Ferguson, 1976, p. 168), was adopted. The results of this analysis are shown in Table 2. YR showed a similar novelty preference to that of the controls for both objects and faces at a $0 \mathrm{~s}$ delay. However, for both types of material she showed a significant difference in novelty preference from the controls at delays of 5 and $10 \mathrm{~s}$.

Additional $t$-tests were carried out to determine whether or not the novelty preference exhibited by YR and the control participants was significantly above chance. These $t$-tests showed that novelty preference in each individual control participant was significantly above chance in each condition (all $P$ 's $<0.001$ ). In contrast, YR's novelty preference was only significantly above chance in the 'objects $0 \mathrm{~s}$ ' condition $(t(11)=2.478, P<0.05)$ and the 'faces $0 \mathrm{~s}$ ' condition $(t(11)$ $=3.144, P<0.01)$. In all other conditions, YR's novelty preference did not differ from chance (all $P$ 's $>0.2$ ). On the DMS task, YR and all control participants had perfect scores.

\section{Discussion}

On the VPC task, YR showed normal novelty preference at a $0 \mathrm{~s}$ delay but was impaired relative to controls, and did not show novelty preference, at increased delays of 5 and $10 \mathrm{~s}$. In contrast YR's item recognition, as tested by the DMS task, which used the same types of object and face stimuli as those used in the VPC task, was unimpaired at delays of up to $10 \mathrm{~s}$. YR correctly recognised all the studied target stimuli, even though she had not shown a novelty preference in the VPC task.

YR's abnormal novelty preference for faces and objects as shown by the VPC task is consistent with previous re- 
sults from humans and non-human primates. In monkeys, a deficit has been observed after a $10 \mathrm{~s}$ delay following a specific lesion of the hippocampus (Zola et al., 2000), or after a lesion of the perirhinal cortex (Buffalo et al., 1999), or after a lesion of the hippocampal formation (Pascalis \& Bachevalier, 1999). A lesion of the area TE led to a deficit without delay (Buffalo et al., 1999).

A human study involving patients with various aetiologies showed that a deficit occurred between $2 \mathrm{~min}$ and a $1 \mathrm{~h}$ delay (McKee \& Squire, 1993). Some of this evidence suggests that hippocampal damage, whether in humans or non-human primates, disrupts VPC performance just as was found in YR. In YR, an abnormality in novelty preference behaviour became apparent at short delays of only $5 \mathrm{~s}$. The temporal characteristics of YR's abnormality are similar to those observed in studies with monkeys (Pascalis \& Bachevalier, 1999; Zola et al., 2000) but appear to differ from those of the amnesic patients reported by McKee and Squire (1993). McKee and Squire's patients showed novelty preference at a 2 min delay but not after $1 \mathrm{~h}$. Although five of McKee and Squire's patients had midline diencephalic lesions, six of them had relatively selective hippocampal lesions so they should be expected to perform like YR. Unfortunately, McKee and Squire did not report the novelty preference scores for individual patients. Although the mean performance of the group $(53.1 \%)$ at the 2 min delay was significantly above chance (50\%), the range of performance (50.6-59.0\%) suggests that there were some individual patients whose novelty preference may not have differed from chance. Furthermore, the mean performance of the amnesic group was significantly impaired relative to the controls (64\%), and represented a $16 \%$ decrease in novelty preference when compared with performance at a $0.5 \mathrm{~s}$ delay $(69.1 \%)$. This can be contrasted with the controls who showed only a $4.1 \%$ decrease in performance from a $0.5 \mathrm{~s}(68.1 \%)$ to a $2 \mathrm{~min}$ delay. Thus, the VPC data from YR are not necessarily inconsistent with those reported by McKee and Squire (1993) as it is possible that some of McKee and Squire's patients may have presented a deficit similar to YR that is masked by averaging.

There remains the puzzle, however, why YR shows an impaired novelty preference despite her ceiling level performance on item recognition using DMS when this was explicitly tested using the same types of materials and task design. It is unlikely that the solution to this puzzle relates to differences in the materials used in the two studies. There is, however, another possible explanation which needs to be considered briefly. Since running this study in 1999 and 2000, YR's performance on a range of tests of intelligence, perception and memory has shown deterioration, and she no longer exhibits the consistent behavioural pattern described above, which she had displayed up until early 1999. She is currently being investigated clinically for a dementing illness, and it is possible that, if confirmed, the disease may have been progressing at the time of VPC testing. However, there was one clear exception to the cognitive decline that became apparent on reassessment in 2000: YR's face recognition appeared to be intact. Moreover, on tests of face recognition that were given over several years from 1995, she performed at slightly above the mean level of her controls and showed no deterioration across time (Mayes et al., 2002). Specifically, her performance on one of these tests, which was modelled on the WRMT and administered 3 months before VPC testing, was normal. We can, therefore, argue that preference for novel faces can be impaired at delays as short as $5 \mathrm{~s}$ in a patient who not only showed face recognition at ceiling levels on a DMS task using comparable delays to the VPC task, but also showed normal performance on a more sensitive, conventional test of face recognition memory.

With respect to YR's preference for novel over familiar objects, we have weaker evidence for a dissociation between impaired VPC performance and intact item recognition. We do not, unfortunately, have any independent item recognition data for YR collected at the time of the VPC testing and, as for the faces, both YR and her controls performed at ceiling levels in the objects DMS task. However, given that the pattern of YR's VPC and DMS performance was similar for the faces and the objects, it remains plausible that a similar dissociation exists for objects between impaired VPC performance and intact item recognition.

The present pattern of results is consistent with a study of nonhuman primates which also indicated a single dissociation between novelty preference and item recognition. Bachevalier et al. (2003) found that adult hippocampallesioned monkeys showed impaired novelty preference at the same delay (60 s) at which DNMS performance was normal. Further work is needed to determine whether other human patients with relatively selective hippocampal lesions resemble YR in showing this single dissociation. Identifying the correct explanation of such a single dissociation will also require further research. Four distinct explanations are worth considering. The first explanation is that YR's VPC impairment reflects an underlying motivational deficit. The second explanation is that the VPC task is more sensitive to deficits in the familiarity memory process that is critical for good visual item recognition than is the DMS task. The third explanation is that some patients with hippocampal damage, such as YR, are able to compensate for their impairments in recollection and familiarity memory by using an alternative strategy. The fourth explanation is that performance on VPC is affected by impairments in recollection.

The first explanation is that our measure of novelty preference (i.e. looking preferentially at the novel item relative to the familiar item) does not reflect recognition memory but instead reflects an underlying motivation to respond to novelty per se. According to this explanation YR shows an impairment on the VPC task because of some underlying motivational deficit. This account seems unlikely for a number of reasons. First, the nature of the VPC task (i.e. passively looking at one picture preferentially over another) 
seems unlikely to have a motivational component. Second, this would not explain why YR's deficit only became apparent after a delay of $5 \mathrm{~s}$; at a delay of $0 \mathrm{~s}$ she showed normal VPC performance for both faces and objects. Third, although hippocampal lesions have been associated with deficits in exploration and curiosity (O'Keefe \& Nadel, 1978), Honey, Watt, \& Good (1998) showed that it is only for novel combinations of stimuli (e.g. a tone and light that had been seen previously, paired not with each other, but with other stimuli) that hippocampally lesioned rats showed a deficit in orienting to novelty; both lesioned and control animals showed a normal orienting response to novel stimuli per se (e.g. a pairing of a tone and light neither of which had been seen previously). This is consistent with O'Keefe and Nadel's (1978) view of genuine novelty which they argue is a reflection of a new configuration of stimuli (which themselves may already be familiar) within an environment. The current experiment does not allow us to make a distinction between detection of, and orienting to, novelty but we propose that YR's deficit does not reflect a motivational deficit.

According to the second explanation, YR shows impaired novelty preference because the VPC test is more sensitive to familiarity memory deficits than the DMS test. This seems implausible given that YR was performing slightly better than her controls' mean scores on face recognition tests that were more demanding than recognising single faces at short delays (Mayes et al., 2002). In other words, YR was impaired at the VPC task, which does not demand effort, whereas she performed better than the mean level of her control participants on demanding face recognition tests. Furthermore, we have directly demonstrated that YR showed completely normal familiarity memory for faces (see Holdstock et al., 2002) although her recall (Mayes et al., 2002) and recollection (Mayes et al., in press) were severely impaired. There is, therefore, good reason to suppose that YR's familiarity memory at least for faces was intact, and that the dissociation between impaired novelty preference and normal DMS performance was not a reflection of task sensitivity.

The third explanation is that some patients with relatively selective hippocampal lesions are aware of their memory deficit and are able to compensate for it by using an alternative strategy, which supports accurate performance on the DMS test and other tests of item recognition, and which does not depend on the integrity of the hippocampus as has been postulated in monkey research (Bachevalier et al., 2003; Ridley \& Baker, 1991). Such an alternative strategy would only be triggered when participants know that their memory is being examined with the DMS test or equivalent recognition tests. The VPC is an incidental memory task so participants would not intentionally activate the compensatory strategy and would, therefore, show a deficit. This explanation implies that familiarity memory would be impaired following hippocampal lesions if one could test recognition in an incidental fashion. This, of course, is not feasible if items are tested one at a time because participants rapidly realise what is happening. Some findings are consistent with amnesics having a problem with recognition when encoding is largely incidental and, therefore, presumably automatic (Mayes, MacDonald, Donlan, Pears, \& Meudell, 1992), but the possibility has not been formally tested in human amnesic patients, and patients with relatively selective hippocampal lesions in particular. If automatic "familiarity memory" processing activates the hippocampus at retrieval as well as at encoding, then YR's VPC deficit could have depended critically on her not realising what the task truly involved throughout the test so that at no stage was she intentionally remembering. Testing this hypothesis will be extremely difficult. It is also implausible for two reasons. First, there are no obvious candidates for the role of intentionally directed compensatory processes that will produce normal familiarity. Second, there is growing evidence that the hippocampus is not engaged in normal people either by encoding that produced subsequent familiarity memory (Davachi, Mitchell, \& Wagner, 2003; Ranganath et al., 2004) or by familiarity memory per se (Eldridge, Knowlton, Furmanski, Bookheimer, \& Engel, 2000).

The fourth explanation is that YR and most other patients with selective hippocampal damage have preserved familiarity memory processes, which are mediated by the perirhinal cortex and other intact extra-hippocampal structures, whether their encoding and retrieval are being intentionally driven or are on "automatic pilot". But there is agreement that such patients have impaired recollection and this also contributes to item recognition performance (e.g. see Mandler, 1980). The VPC is a task that depends on participants detecting a difference in familiarity between two stimuli and orienting towards the more novel stimulus. Use of the $c$-fos technique in rats has demonstrated that the perirhinal cortex and area TE are activated by novel visual items, but not novel visuo-spatial combinations of familiar items, whereas the hippocampus is activated by novel visuo-spatial combinations of familiar items, but not by novel items (Wan, Aggleton, \& Brown, 1999). This is consistent with the hippocampus not playing a direct role in the detection of novel items. YR's impaired VPC performance suggests that she was reacting to her impaired recollection in the face of normal familiarity memory for the faces and objects. Thus, although the novel items might have been drawing her attention so might the familiar ones because she was unable to recall why they were familiar. The result would have been the absence of preference for the novel items at the longer delays used in the VPC task. Presumably, at the shortest delay $(0 \mathrm{~s})$ YR was still able to recollect why one of a pair of faces was familiar. One implication of this prediction is that normal participants may show reduced novelty preferences when the competing stimulus is familiar, but not recollected. If this is found, then VPC performance will have been shown to be sensitive to mismatches between recollection and familiarity. This is consistent with the view that hippocampal damage disrupts recollection and recall, but not item familiarity memory. 


\section{Acknowledgements}

The authors would like to thank Jim Stone for helpful discussions.

\section{References}

Aggleton, J. P., \& Brown, M. W. (1999). Episodic memory, amnesia, and the hippocampal-anterior thalamic axis. Behavioural and Brain Sciences, 22, 425-489.

Alvarez, P., Zola-Morgan, S., \& Squire, L. R. (1995). Damage limited to the hippocampal region produces long-lasting memory impairment in monkeys. Journal of Neuroscience, 15, 3796-3807.

Bachevalier, J., Nematic, S., \& Alvarado, M. C. (2003). The medial temporal structures and object recognition memory in nonhuman primates. In L. R. Squire \& D. L. Schacter (Eds.), Neurospsychology of Memory (3rd ed.), (pp. 326-338). Guilford Press.

Bachevalier, J., Beauregard, M., \& Alvarado, M. C. (1999). Long-term effect of neonatal damage to the hippocampal formation and amygdaloid complex on object discrimination and object recognition in rhesus monkey (Macaca mulatta). Behavioral Neuroscience, 113, $1127-1151$.

Bachevalier, J., Brickson, M., \& Hagger, C. (1993). Limbic-dependent recognition memory in monkeys develops early in infancy. NeuroReport, 4, 77-80.

Baddeley, A., Emslie, H., \& Nimmo-Smith, I. (1994). Doors and People. Thames Valley Test Company.

Baxter, M. G., \& Murray, E. A. (2001a). Impairments in visual discrimination learning and recognition memory produced by neurotoxic lesions of rhinal cortex in rhesus monkeys. European Journal of Neuroscience, $13,1228-1238$.

Baxter, M. G., \& Murray, E. A. (2001b). Opposite relationship of hippocampal and rhinal cortex damage to delayed nonmatching-tosample deficits in monkeys. Hippocampus, 11, 61-71.

Buffalo, E. A., Ramus, S. J., Clark, R. E., Teng, E., Squire, L. R., \& Zola, S. M. (1999). Dissociation between the effects of damage to perirhinal cortex and area TE. Learning and Memory, 6, 572-599.

Cipolotti, L., Shallice, T., Chan, D., Fox, N., Scahill, R., \& Harrison, G. et al., (2001). Long-term retrograde amnesia the crucial role of the hippocampus. Neuropsychologia, 39, 151-172.

Davachi, L., Mitchell, J. P., \& Wagner, A. D. (2003). Multiple routes to memory: distinct medial temporal lobe processes build item and source memories. Proceedings of the National Academy of Sciences of the United States of America, 100, 2157-2162.

Eldridge, L. L., Knowlton, B. J., Furmanski, C. S., Bookheimer, S. Y., \& Engel, S. A. (2000). Remembering episodes: a selective role for the hippocampus during retrieval. Nature Neuroscience, 3, 1149-1152.

Fagan, J. F. (1974). Infant recognition memory: the effects of length of familiarization and type of discrimination task. Child Development, $45,351-356$.

Fantz, R. L. (1964). Visual experience in infants: decreased attention to familiar patterns relative to novel ones. Science, 146, 668-670.

Ferguson, G. A. (1976). Statistical Analysis in Psychology and Education (4th ed.). Tokyo: McGraw-Hill.

Gaffan, D., \& Murray, E. A. (1992). Monkeys (Macaca fascicularis) with rhinal cortex ablations succeed in object discrimination learning despite 24-h intertrial intervals and fail at matching to sample despite double sample presentations. Behavioural Neuroscience, 106, 30-38.

Holdstock, J. S., Mayes, A. R., Cezayirli, E., Isaac, C. L., Aggleton, J. P., \& Roberts, N. (2000). A comparison of egocentric and allocentric spatial memory in a patient with selective hippocampal damage. Neuropsychologia, 38, 410-425.

Holdstock, J. S., Mayes, A. R., Roberts, N., Cezayirli, E., Isaac, C. L., \& O'Reilly, R. C. et al., (2002). Under what conditions is recognition spared relative to recall after selective hippocampal damage? Hippocampus, 12, 341-351.
Honey, R. C., Watt, A., \& Good, M. (1998). Hippocampal lesions disrupt an associative mismatch process. Journal of Neuroscience, 18, 22262230.

Mandler, G. (1980). Recognizing: the judgement of previous occurrence. Psychological review, 87, 252-271.

Manns, J. R., \& Squire, L. R. (1999). Impaired recognition memory on the Doors and People Test after damage limited to the hippocampal region. Hippocampus, 9, 495-499.

Manns, J. R., Stark, C. E. L., \& Squire, L. R. (2000). The visual paired-comparison task as a measure of declarative memory. Proceedings of the National Academy of Sciences of the United States of America, 97, 12375-12379.

Manns, J. R., Hopkins, R. O., Reed, J. M., Kitchener, E. G., \& Squire, L. R. (2003). Recognition memory and the human hippocampus. Neuron, $37,171-180$.

Mayes, A. R., MacDonald, C., Donlan, L., Pears, J., \& Meudell, P. R. (1992). Amnesics have a disproportionately severe memory deficit for interactive context. Quarterly Journal of Experimental Psychology, 45A, 265-297.

Mayes, A. R., Holdstock, J. S., Isaac, C. L., Hunkin, N. M., \& Roberts, N. (2002). Relative sparing of item recognition memory in a patient with adult-onset damage limited to the hippocampus. Hippocampus, $12,325-340$.

Mayes, A. R., Holdstock, J. S., Isaac, C. L., Montaldi, D., Grigor, J., \& Gummer, A. et al., in press. Associative recognition in a patient with selective hippocampal lesions and relatively normal item recognition. Hippocampus.

McKee, R. D., \& Squire, L. R. (1993). On the development of declarative memory. Journal of Experimental Psychology: Learning, Memory and Cognition, 19, 397-404.

Meunier, M., Bachevalier, J., Mishkin, M., \& Murray, E. A. (1993). Effects on visual recognition of combined and separate ablations of the entorhinal and perirhinal cortex in rhesus monkey. Journal of Neuroscience, 13, 5418-5432.

Murray, E. A., \& Mishkin, M. (1998). Object recognition and location memory in monkeys with excitotoxic lesions of the amygdala and hippocampus. Journal of Neuroscience, 5, 68-82.

Nelson, C. A. (1995). The ontogeny of human memory: a cognitive neuroscience perspective. Developmental Psychology, 3, 723-738.

Nelson, H. E. (1991). National Adult Reading Test (2nd ed.). London: NFER-Nelson.

O'Keefe, J. \& Nadel, L. (1978). The Hippocampus as a Cognitive Map. Oxford University Press.

Pascalis, O., \& Bachevalier, J. (1998). Face recognition in primates: a cross species study. Behavioural Processes, 43, 87-96.

Pascalis, O., \& Bachevalier, J. (1999). Neonatal aspiration lesions of the hippocampal formation impair visual recognition memory when assessed by paired-comparison task but not by delayed nonmatching-tosample task. Hippocampus, 9, 609-616.

Pascalis, O., de Haan, M., Nelson, C. A., \& de Schonen, S. (1998). Long-term recognition memory for faces assessed by visual paired comparison in 3- and 6-month-old infants. Journal of Experimental Psychology: Learning, Memory and Cognition, 24, 249-260.

Ranganath, C., Yonelinas, A. P., Cohen, M. X., Dy, C. J., Tom, S. M., \& D'Esposito, M. (2004). Dissociable correlates of recollection and familiarity within the medial temporal lobes. Neuropsychologia, 42, $2-13$.

Reed, J. M., \& Squire, L. R. (1997). Impaired recognition memory in patients with lesions limited to the hippocampal formation. Behavioural Neuroscience, 111, 667-675.

Ridley, R. M., \& Baker, H. F. (1991). A critical evaluation of monkey models of amnesia and dementia. Brain Research Review, 16, 15-37.

Squire, L. R. (1992). Declarative and nondeclarative memory: multiple brain systems supporting learning and memory. Journal of Cognitive Neuroscience, 4, 232-243.

Vargha-Khadem, F., Gadian, D. G., Watkins, K. E., Connelly, A., Van Paesschen, W., \& Mishkin, M. (1997). Differential effects of early 
hippocampal pathology on episodic and semantic memory. Science, 277, 376-380.

Wan, H., Aggleton, J. P., \& Brown, M. W. (1999). Different contributions of the hippocampus and perirhinal cortex to recognition memory. The Journal of Neuroscience, 19, 1142-1148.

Warrington, E. K. (1984). Recognition Memory Test. London: NFERNelson.

Warrington, E. \& James, M. (1991). Visual Object and Space Perception Battery. Thames Valley Test Company.

Wechsler, D. (1981). Wechsler Adult Intelligence Scale, Revised. New York: Psychological Corporation.

Wechsler, D. (1987). Wechsler Memory Scale, Revised. New York: Psychological Corporation.

Yonelinas, A. P., Kroll, N. E., Quamme, J. R., Lazzara, M. M., Sauve, M. J., \& Widaman, K. F. et al., (2002). Effects of extensive temporal lobe damage or mild hypoxia on recollection and familiarity. Nature Neuroscience, 5, 1236-1241.

Zola, S. M., \& Squire, L. R. (2001). Relationship between magnitude of damage to the hippocampus and impaired recognition memory in monkeys. Hippocampus, 11, 92-98.

Zola, S. M., Squire, L. R., Teng, E., Stefanacci, L., Buffalo, E. A., \& Clark, R. E. (2000). Impaired recognition memory in monkeys after damage limited to the hippocampal region. Neuroscience, 20, 451-463.

Zola-Morgan, S., Squire, L. R., Amaral, D. G., \& Suzuki, W. A. (1989). Lesions of perirhinal and parahippocampal cortex that spare the amygdala and hippocampal formation produce severe memory impairment. Journal of Neuroscience, 9, 4355-4370.

Zola-Morgan, S., Squire, L. R., Rempel, N. L., Clower, R. P., \& Amaral, D. G. (1992). Enduring memory impairment in monkeys after ischemic damage to the hippocampus. Journal of Neuroscience, 12, 2582-2596. 